\title{
EHMTI-0398. Long term safety of the ATI neurostimulation system for the treatment of cluster headache
}

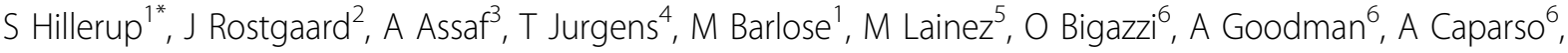 \\ R Jensen ${ }^{1}$, A May $^{4}$
}

From 4th European Headache and Migraine Trust International Congress: EHMTIC 2014

Copenhagen, Denmark. 18-21 September 2014

\section{Introduction}

At least two-thirds of cluster headache $(\mathrm{CH})$ patients that received the ATI Neurostimulation System have achieved profound clinical improvements including acute headache pain relief and/or significant attack frequency reduction. The ATI Neurostimulator is inserted trans-orally using a minimally invasive technique.

\section{Aim}

This analysis aims to characterize the long term safety of the ATI Neurostimulation System in $\mathrm{CH}$ sufferers.

\section{Method}

Patients from the Pathway CH-1 and Pathway R-1 studies were included in the analysis. All adverse events (AEs) including transient swelling and pain, were documented and assessed for relationship to procedure and/or the presence of the neurostimulator.

\section{Results}

Ninety-eight (98) patients received the ATI Neurostimulator as of May 2014. Fifteen patients (15\%) reported no AEs and 83 patients (85\%) reported at least one related AE. In total, $341 \mathrm{AEs}$ were reported (average 4.1 AEs/ patient). Currently, 216 (63\%) of all AEs have resolved; average time to resolution was 69 days (range 0-611). The majority of reported AEs (77.4\%) occurred within 30 days of the insertion procedure (peri-op AEs). Of these AEs, $82 \%$ of patients experienced sensory disturbances. The large majority (72\%) of these events had a mild to moderate impact on the patient's daily activities and were transient, with an average resolution of 110.3 days (range 20-313).

\section{Conclusion}

The majority of AEs were reported within 30 days of the Neurostimulator insertion procedure and the majority resolved within 3 months. These AEs are not different from standard sequelae reported for other trans-oral procedures and display a similar time course for healing.

Conflict of interest.

\section{Authors' details}

'Danish Headache Center, Glostrup Hospital University of Copenhagen, Copenhagen, Denmark. ${ }^{2}$ Department of Oral and Maxillofacial Surgery, Rigshospitalet University of Copenhagen, Copenhagen, Denmark. ${ }^{3}$ Department of Oral and Maxillofacial Surgery, Universitätsklinikum Hamburg-Eppendorf, Hamburg, Germany. ${ }^{4}$ Department of Neurology, Universitätsklinikum Hamburg-Eppendorf, Hamburg, Germany. ${ }^{5}$ Department of Neurology, Hospital Clinico Universitario Universidad de Valencia, Valencia, Spain. ${ }^{6}$ Clinical Affairs, Autonomic Technologies Inc., Redwood City, USA.

Published: 18 September 2014

doi:10.1186/1129-2377-15-S1-14

Cite this article as: Hillerup et al:: EHMTI-0398. Long term safety of the ATI neurostimulation system for the treatment of cluster headache. The Journal of Headache and Pain 2014 15(Suppl 1):I4. 